\title{
COMUNICACIÓN
}

\section{Ingreso y evolución del parasitismo por Melophagus ovinus en una majada Corriedale en el noroeste de la Patagonia Argentina}

\author{
FERMÍN OLAECHEA*, JUAN CORLEY*, MARCELA LARROZA,* \\ FERNANDO RAFFO* y RAÚL CABRERA*
}

\section{DISSEMINATION OF SHEEP KED (Melophagus ovinus) WITHIN A NON INFESTED CORRIEDALE FLOCK IN NW PATAGONIA, ARGENTINA}

An infested ram was introduced during mating (for 2 weeks) into a non-infested Corriedale sheep flock. The rate at which keds were disseminated, was measured by tallying number of infected sheep and counting ked burden on days 0, 220 and 433. To evaluate potential growth of Melophagus ovinus populations within the fixed-sized sheep flock during the study period, we built a simple population model. Initial counts (day 0), were of $4 \mathbf{M}$. ovinus adults and 3 pupae on a single sheep, whereas on day 220 parasitism reached $100 \%$ in lambs remaining at these values throughout the study period. In contrast, adult sheep showed low but rising counts, reaching $91 \%$ on day 433. According to the model, expected M. ovinus populations were 193 fold larger. We assign this difference to limitations on population growth set by environmental factors and sheep management such as shearing, lambing and lamb sales.

Key words: Melophagus ovinus, sheep, sheep ked, epidemiology.

\section{INTRODUCCIÓN}

Melophagus ovinus, conocido como "falsa garrapata" en nuestro país, es un insecto de la Familia Hippoboscidae (díptero áptero), que hace vida parasitaria en forma permanente y obligada principalmente en ovinos. Toda su vida transcurre en el vellón, y la transmisión ocurre por contacto directo entre los animales ${ }^{1}$. El ciclo biológico tiene una duración variable de 24 a 36 días ${ }^{1,2}$, relativamente largo en comparación con los ciclos de otros ectoparásitos de los lanares. Es hematófago estricto, y la sangre constituye su única ingesta ${ }^{3}$.

El M. ovinus es uno de los parásitos más cosmopolitas y frecuentes de los ovinos de distintos países, sobre todo de las áreas templadas y frías. La falta de control oficial y los cambios en el manejo de las majadas permitirían un aumento de su prevalencia ${ }^{4,5}$. En la Patagonia Argentina, su hallazgo ha sido constante en las zonas húmedas de la precordillera y sur de Santa Cruz y Tierra del Fuego, pero en los últimos años, aunque es una parasitosis de denuncia obligatoria y existen exigencias oficiales para su control (Res. 197/78 de SENASA), se ha registrado una dispersión que también afecta las majadas de la meseta árida y costa atlántica, estimándose que el $70 \%$ de las majadas

* National Institute for Agricultural Technology (INTA), CC 277, (8400) Bariloche, Argentina. folaechea@bariloche.inta.gov.ar 
patagónicas están afectadas por melófagos ${ }^{6}$.

Si bien existe información bibliográfica sobre la dinámica poblacional y algunas características biológicas del melófago, donde se registran variaciones relacionadas con las distintas condiciones climáticas, la dinámica poblacional de este parásito no ha sido estudiada en Sudamérica.

Este trabajo describe una situación a campo donde, desde el ingreso del melófago a una majada libre, se observa la evolución de la población parasitaria. Los resultados de estas observaciones fueron utilizados para la elaboración de un modelo de crecimiento poblacional simple con el que se pudo estimar la curva potencial de crecimiento del parásito y compararla con los datos observados a campo.

\section{MATERIAL Y MÉTODOS}

El trabajo se realizó en un establecimiento de la cordillera rionegrina $\left(41^{\circ} 05^{\prime} \mathrm{L}\right.$. S., $71^{\circ} 31^{\prime} \mathrm{L}$. O.), donde $M$. ovinus se introdujo accidentalmente por un carnero infectado que permaneció con la majada durante 2 semanas. A partir de ese momento, no se realizó ningún tratamiento antiparasitario con el objetivo de estudiar la evolución de M. ovinus en la majada.

La población en estudio estaba conformada por 166 ovinos cruza Corriedale. Las categorías presentes durante las observaciones fueron: corderos, borregos, ovejas y carneros, para el análisis de los datos se diferenciaron en corderos (menores de un año, $n=60$ ) y en ovinos adultos $(n=106)$. Los conteos de parásitos se realizaron a partir del diagnóstico de la infección (día 0) y los días 220 y 433.

Los animales, identificados con caravanas, permanecieron bajo el manejo habitual de la zona en potreros con pasturas naturales y encierros nocturnos.

En cada control se registró el número de melófagos en animales elegidos al azar, en no menos del 50\% del total de la majada. El conteo de parásitos se realizó de acuerdo a Nelson ${ }^{7}$, con el animal sujeto, echado sobre el flanco izquierdo y revisando desde la cabeza hasta la cola todo el lado derecho del animal. El número de melófagos obtenido se multiplicó por 2 y de esta manera se calculó el número total de melófagos por ovino. En caso de no encontrar melófagos en el lado derecho, se buscó cuidadosamente en el lado izquierdo, para de esta manera determinar el 0 (cero) como no presencia de parásitos en toda la superficie corporal del animal.

\section{Modelo de simulación}

Para evaluar el crecimiento potencial de $M$. ovinus durante el período de estudio se construyó un modelo de simulación ${ }^{8}$, basado en observaciones realizadas en corderos desde el día 0 al día 220 . La ecuación utilizada es:

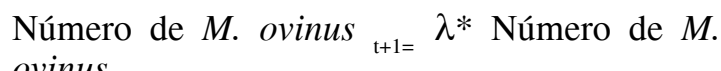
ovinus $_{\mathrm{t}}$

donde $\lambda$ es la tasa de crecimiento poblacional, que fluctúa asumiendo una Función de Densidad de Probabilidades (FDP) con distribución normal de media $\mu=\lambda=2,15$. Se asume que no existe densidad-dependencia en $\lambda$ y un tiempo generacional de 32 días $^{4}$.

\section{RESULTADOS}

A partir del hallazgo inicial (día 0) de una oveja con 4 melófagos y 3 pupas, al día 220 se registró la presencia del parasitismo en el 100\% de los corderos revisados que en ese momento tenían 6 meses de edad. Ese mismo porcentaje de infestación se registró en la nueva generación de corderos que formaron parte del conteo posterior, el día 433, cuando contaban aproximadamente con 3 meses de edad. Por otro lado, en los ovinos adultos, el porcentaje de infección fue aumentando lentamente hasta llegar al $91 \%$ el día 433. Las mayores infestaciones fueron observadas en corderos los días 220 y 433, mostrando medias de 13,4 y 35,7 melófagos y valores máximos de 37 y 69 respectivamente. Los ovinos adultos tuvieron bajos conteos, con medias 2,4 y 6,3, y valores máximos de 16 y 33 en los días 220 y 433 respectivamente (Figura $1)$.

El modelo de simulación (Figura 2), muestra que durante la etapa de colonización (hasta el día 220), las poblaciones de M. ovinus crecieron de modo exponencial. En el día 433, el valor calculado en la curva de crecimiento potencial fue de 825.186 melófagos, aproximadamente 193 veces mayor al valor medido en los corderos en la misma fecha, que fue de 4.284 melófagos. 

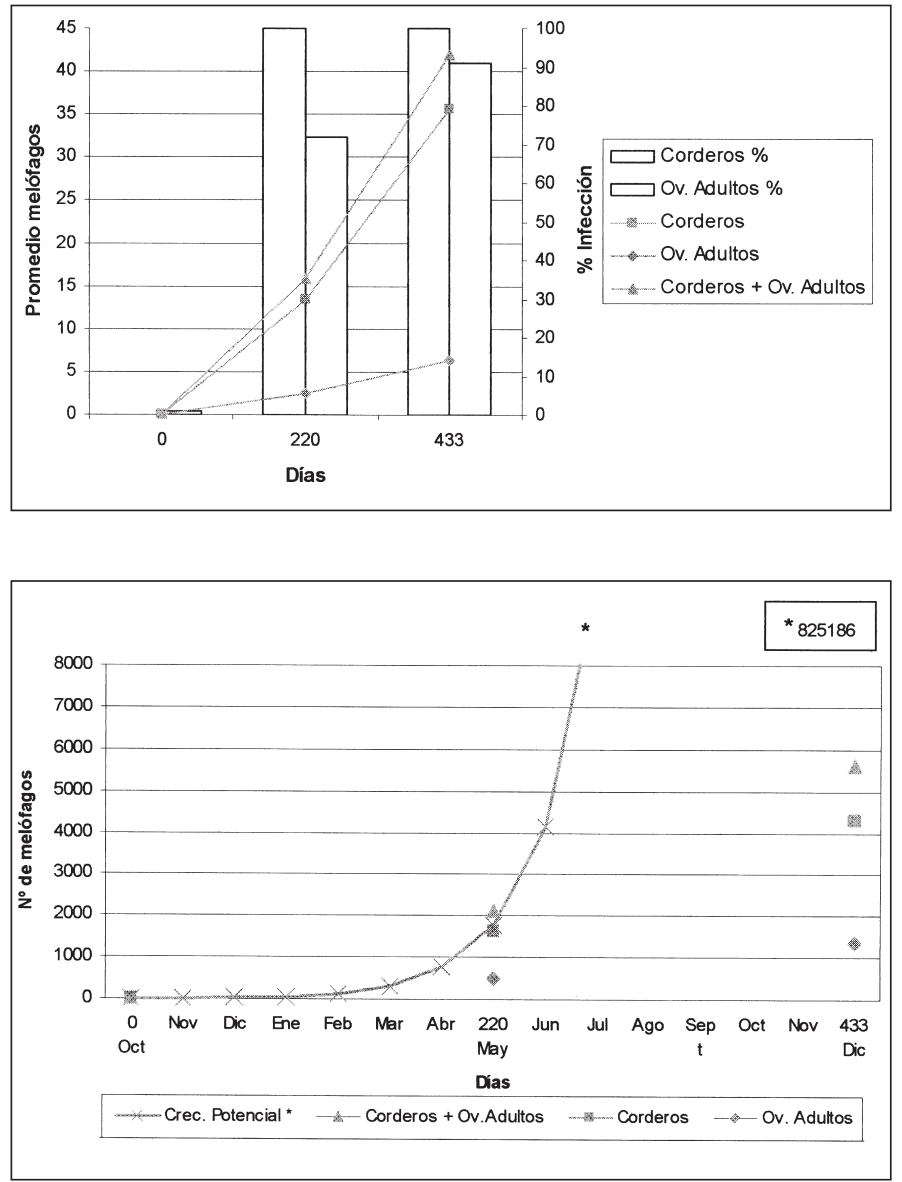

Figura 1. Promedio del conteo de melófagos y porcentaje de ovinos infectados (adultos y corderos) observados en corderos y ovinos adultos, los días 0,220 y 433.
Figura 2. Total de melófagos hallados en los días 0,220 y 433 en ovinos (adultos y corderos) y curva de crecimiento potencial (línea sólida) de la población de melófagos.

\section{DISCUSIÓN}

Posteriormente, al ingreso del parásito a la majada, la evolución fue lenta y con bajos conteos por animal, teniendo la máxima expresión en los corderos, que en el día 220 registran el 100\% de afectados y con conteos de más de 5 veces superiores a los ovinos adultos, a los que se les atribuye capacidad de resistencia a este ectoparásito $^{9,10}$. El mayor hallazgo de la carga parasitaria en los corderos es coincidente con el hallazgo de $\mathrm{Pdaf}^{11}$, y fue adjudicada tanto a la migración de parásitos desde las ovejas madres como a una mayor tasa de reproducción de los melófagos sobre esta categoría, considerada más sensible.

Estudios previos sobre dinámica poblacional ${ }^{1,11-}$ ${ }^{14}$, muestran incrementos de la carga parasitaria en el invierno, primavera y otoño, con disminuciones durante el verano. En la majada en estudio se asume que además de las temperaturas, la población parasitaria fue también influenciada por las maniobras convencionales de manejo tales como esquila (la cual determina una pérdida de hasta el $97 \%$ de los parásitos en el vellón ${ }^{15}$ ), venta de corderos (que portan las mayores cargas de parásitos ${ }^{16,15}$ ) y pariciones (aparición de nuevos huéspedes que reciben melófagos de los adultos). En esta suma de factores, condicionantes de la abundancia y estacionalidad de las poblaciones de melófagos, se explicaría la gran diferencia observada entre la capacidad potencial de crecimiento parasitario y la carga moderada a baja hallada en esta majada al día 433 (Figura 2). El cálculo del potencial de crecimiento estimula a proponer seguimientos de las poblaciones de melófagos en ovinos en diferentes ambientes y manejos, ya que los datos de majadas con altas cargas de melófagos son comunes en distintas zonas de la Patagonia. 
Es de destacar que si bien fueron descriptos síntomas asociados a la presencia de melófagos, tales como prurito, rascado y mordeduras en los animales como respuesta a la irritación ${ }^{4}$, en este trabajo no fueron observados. En los manejos extensivos, este tipo de parasitismo asintomático retrasa el diagnóstico hasta el momento de junta $\mathrm{y}$ esquila, donde en general se subestiman las poblaciones parasitarias pues no se controla el cordero y los ovinos esquilados (mayores de 1 año), tienen muy bajas cargas o no están todos afectados. Se asume que la situación descripta es la frecuente en establecimientos cordilleranos con manejos similares, siendo en general a partir del segundo año del ingreso del melófago a la majada cuando es visualizada como problema por el productor.

\section{RESUMEN}

Un carnero infestado con $M$. ovinus fue introducido durante dos semanas en una majada de ovinos cruza Corriedale que se encontraba libre del parásito. La evolución de M. ovinus fue medida registrando los ovinos infestados y realizando conteos de parásitos sobre los animales en los días 0, 220 y 433. Los resultados de estas observaciones fueron utilizados para la elaboración de un modelo de crecimiento poblacional simple con el que se pudo estimar la curva potencial de crecimiento del parásito y compararla con los datos observados a campo. El hallazgo inicial de parasitismo, el día 0 , fue de 4 melófagos y 3 pupas en una oveja de la majada; el día 220 se registró la presencia de parasitismo en el $100 \%$ de los corderos y ese mismo porcentaje de infestación se observó en la siguiente generación de corderos revisados el día 433. Por otro lado, en los ovinos adultos las cargas parasitarias fueron menores y el porcentaje de infección fue aumentando lentamente hasta llegar al $91 \%$ el día 433. En esta fecha, la capacidad potencial de crecimiento de la población de melófagos, calculada por el modelo, fue aproximadamente 193 veces mayor al valor medido en los corderos en la misma fecha. La diferencia observada se atribuye a factores ambientales como las temperaturas y a las maniobras convencionales de manejo tales como esquila, venta de corderos y pariciones, que condicionan la abundancia y estacionalidad de las poblaciones de melófagos.

\section{REFERENCIAS}

1.- EVANS G O. Studies on the bionomics of sheep ked, Melophagus ovinus L., in west Wales. Bull Ent Res 1950; 40: 459-78.

2.- NELSON W A, QUALLY M C. Annual cycles in numbers of the sheep ked, Melophagus ovinus (L). Can J Anim Sci 1958; 38: 194-9.

3.- BULMAN G M, LAMBERTI J C. Melophagus ovinus. Manual Técnico AAPAVET. 2001; 90 p.

4.- SMALL R W. A review of Melophagus ovinus (L.), the sheep ked. Veterinary Parasitology 2005; 130: 141-55.

5.- COLEBROOK E, WALL R. Ectoparasites of livestock in Europe and the Mediterranean region. Vet Parasitol 2004; 120: 251-74.

6.- CROVETTO H. Proyecto Nacional de Erradicación de la Melofagosis. Dirección Nacional de Sanidad Animal. SENASA. 2001; 15 p.

7.- NELSON W A, SLEN S B, BANKY E C. Evaluation of methods of estimating populations of the sheep ked, Melophagus ovinus (L.) (Diptera: Hippoboscidae), on mature ewes and young lambs. Can J Anim Sci 1957; 37: 8-13.

8.- GILLMAN M, HAILS R. An introduction to Ecological Modelling. Blackwell Science. 1997; 202 p.

9.- NELSON W A, KOZUB C. Melophagus ovinus (Diptera: Hippoboscidae): evidence of local mediation in acquired resistance of sheep to keds. J Med Entomol 1980; 17: 291-7.

10.- ALLEN J R, NELSON W A. Inmunological responses to ectoparasites. Fortschritte der Zoologie. Band 27, Zbl Bakt. 1982; Suppl. 12.

11.- PFADT R E. Sheep ked populations on a small farm. J Econ Ent 1976; 69: 313-6.

12.- GRAHAM N P H, TAYLOR K L. Studies on the ectoparasites of sheep and their control. I. Observations on the bionomics of the sheep ked Melophagus ovinus. Austr. Council Sci. Ind. Pamphl. 1941; 108: 9-26.

13.- MACLEOD J. The distribution and dynamics of ked populations, Melophagus ovinus Linn. Parasitology 1948; 39: 61-8.

14.- LEGG D E, KUMAR R, WATSON D W, LLOYD J E. Seasonal movement and spatial distribution of the sheep ked (Diptera: Hippoboscidae) on Wyoming lambs. J Econ Entomol 1991; 84: 1532-9.

15.- OLAECHEA F V, CORLEY J. Ked (Melophagus ovinus) transmission: burden on lambs from affected flocks and remnant populations after shearing. 19th. International Conference, World Association for the Advancement of Veterinary Parasitology. New Orleans, EEUU. 2003.

16.- CARBALLO M V, FERNÁNDEZ S. Tratamiento de la piojera ovina en la esquila. Revista del Plan Agropecuario 2002; 99: 4. 